\title{
POSSIBLE SOLAR CYCLE VARIATIONS IN THE CONVECTION ZONE
}

\author{
Sarbani Basu \\ Institute for Advanced Study, Olden Lane, Princeton NJ 08540, U. S. A., and \\ Astronomy Department, Yale University, P.O. Box 208101 New Haven, CT \\ 06520-8101 USA \\ H. M. Antia \\ Tata Institute of Fundamental Research, Homi Bhabha Road, Mumbai 400005, \\ India
}

October 31, 2018

\begin{abstract}
Using data from the Global Oscillations Network Group (GONG) that covers the period from 1995 to 1998 we study the change in frequencies of solar oscillations with solar activity. From these frequencies we attempt to determine any possible variation in solar structure with solar activity. We do not find any evidence of a change in the convection zone depth or extent of overshoot below the convection zone during the solar cycle.
\end{abstract}

Keywords: Sun: General - Sun: Interior - Sun: Oscillations

\section{Introduction}

It is well known that frequencies of solar oscillations vary with time (Elsworth et al. 1990; Libbrecht and Woodard 1990; Dziembowski et al. 1998; Bhatnagar, Jain and Tripathy 1999; Howe, Komm and Hill 1999) and that these variations are correlated with solar activity. To a first approximation the scaled frequency differences between frequencies at different phases of the solar cycle appear to be a smooth function of frequency indicating that the solar-cycle related changes are predominantly taking place near the solar surface. The seat of the solar dynamo, however, is believed to be near the base of the convection zone (hereafter CZ) and thus one may expect some changes in this region during the solar cycle. Monteiro, Christensen-Dalsgaard, and Thompson (1998) have found some hint of variation in extent of overshoot below the solar convection zone. This needs to be confirmed by independent analysis. Thus in this work we investigate whether these frequency differences with time imply any change in depth of the solar convection zone or extent of overshoot with solar activity. 


\section{Data used}

We have used monthly $m$-averaged power spectra from the Global Oscillations Network Group (GONG) project to determine solar oscillation frequencies. We have fitted the rotation corrected, $m$-averaged, power spectra for each GONG month, 1 to 37. Each GONG month extends over 36 days, with month 1 beginning on 7 May, 1995 and month 37 ending on 28 December, 1998. To improve the signal-tonoise ratio in the spectra used to determine the $\mathrm{CZ}$ depth and the extent of overshoot below the CZ, we have summed spectra for four months. We select 19 summed spectra covering the entire period and solar activity range. There is some overlap in the time period covered by these spectra. We have also summed the spectra for GONG months 7 to 22 and 24 to 35 to improve the signal-to-noise ratio still further. The months 7 to 22 cover a period around minimum solar activity extending from 9 December, 1995 to 6 July, 1997, while months 24 to 35 cover a period of high activity from 12 August, 1997 to 17 October, 1998. These two summed spectra provide precise data during period of low and high activity, thus allowing us to measure small variations in solar interior.

To calculate the frequencies from the $m$-averaged power spectra we fit each mode separately using a maximum likelihood technique (Anderson, Duvall and Jefferies 1990). The fit is performed over a region extending midway to the next mode on either side of the peak and includes all leaks which occur within the fitting interval from modes of degree $\ell-3$ to $\ell+3, \ell$ being the degree of the target mode. Because of possibly incorrect splitting coefficients used in constructing the $m$-averaged power spectra the apparent widths of the peaks may be increased, but that is not likely to affect the fitted frequencies as the width of each leak is treated as a separate parameter while fitting the spectra. Further, in this paper we are interested in the frequency shift with solar cycle so any possible systematic error introduced in fitting the frequencies for an individual month will tend to get cancelled while taking the frequency differences between two months. Moreover we have tested for possible systematic errors in fitting $m$-averaged spectra by comparing the fitted frequencies with those computed by the GONG project using the individual $m$ spectra for the same period (Basu and Antia, 2000). Systematic difference between these two sets of frequencies is $\lesssim 10 \mathrm{nHz}$.

In addition we also use the frequencies from Big Bear Solar Observatory (BBSO) data for the years 1986, 1988, 1989 and 1990. These data sets cover the rising phase of the cycle 22 (Woodard and Libbrecht 
1993). The estimated error in frequencies in these data sets is much larger than those in GONG data.

\section{Technique}

The temperature gradient within the Sun changes from the adiabatic value inside the convection zone to the radiative value below the base of the convection zone. This sharp change in the temperature gradient introduces a bump in the sound speed difference between two models (or between a model and the Sun) if they have different CZ depths. This signal can be used to determine the depth of the CZ. We use the method described by Basu and Antia (1997) to determine the radial position of the CZ base.

The transition of the temperature gradient from the adiabatic to radiative values at the base of the solar convection zone also gives rise to an oscillatory signal in frequencies of all modes which penetrate below the base of the solar convection zone (Gough 1990) and this signal can be used to determine the extent of overshoot below the solar CZ (Monteiro, Christensen-Dalsgaard and Thompson 1994; Basu, Antia and Narasimha 1994). This signal can be amplified by taking the fourth differences of the frequencies as a function of the radial order $n$ enabling a more precise measurement of the amplitude, $A$, of oscillations. This amplitude increases with the extent of overshoot and can be calibrated against amplitudes for models with known extents of overshoot. The frequency $\tau$ of the signal is a measure of the acoustic depth at which

the transition occurs. We use the method described in Basu (1997) to isolate the oscillatory signal and measure its characteristics.

\section{Results}

For each of these summed spectra we fit symmetric Lorentzian profiles to calculate the frequencies using a maximum likelihood approach. Fig. 1 shows the scaled differences in frequencies obtained from months 1-4, 8-11, 26-29 and 32-35 and those obtained from summed spectra for months 7 to 22 . The differences have been averaged in groups of 10 modes to reduce scatter. The frequencies are scaled by the quantity $Q_{n l}$ which is the ratio of the mode inertia of a mode of degree $l$ order $n$ to that of a mode of degree 0 with the same frequency as the mode of degree $l$ order $n$ (Christensen-Dalsgaard and Berthomieu 1991). Note that to a first approximation the shift in frequencies is a smooth function of frequency. We therefore may not expect much change in the deeper 


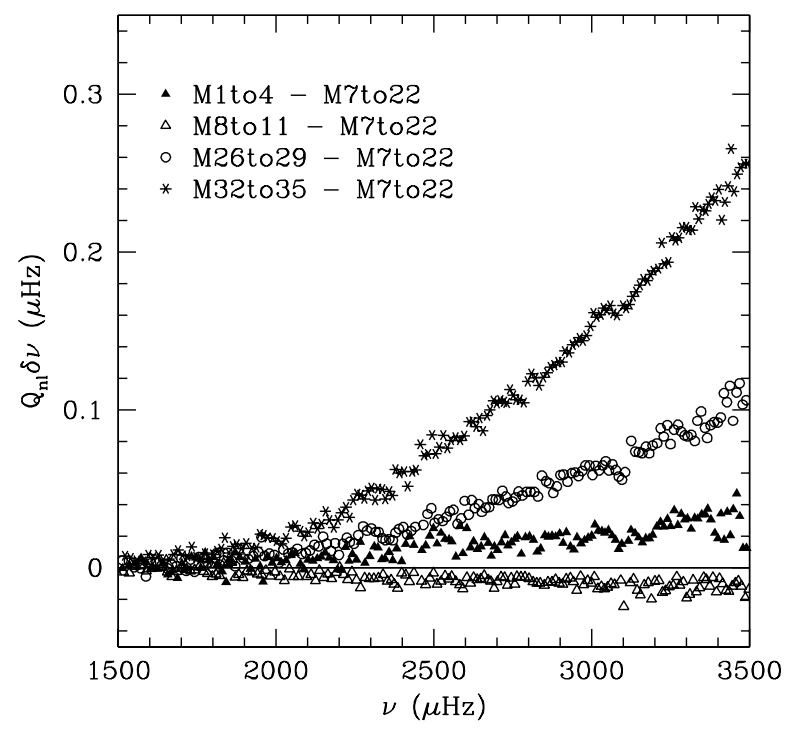

Figure 1. Differences in frequencies determined from observations taken at different times during the solar cycle. M7to22 is the data set for months 7 to 22 , similarly M1to4, M8to11, M26to29 and M32to35 are the data sets covering months 1-4, 8-11, 26-29 and 32-35 respectively. The scaled frequency differences have been averaged in groups of 10 modes to reduce scatter.

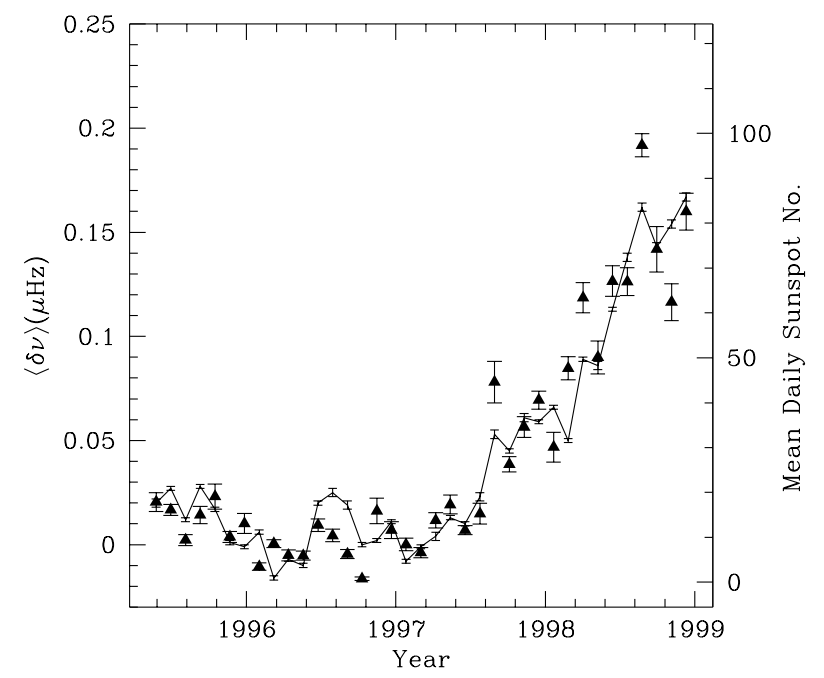

Figure 2. The mean frequency shift averaged over all modes with $1.5 \leq \nu \leq 3.5$ $\mathrm{mHz}$ and $5 \leq \ell \leq 100$ plotted as a function of time for the 37 GONG months is shown by the set of points which are joined by a continuous line. The crosses show the average number of sunspots each day during the period when the observations were taken, the scale for this is on the right-hand axis. 


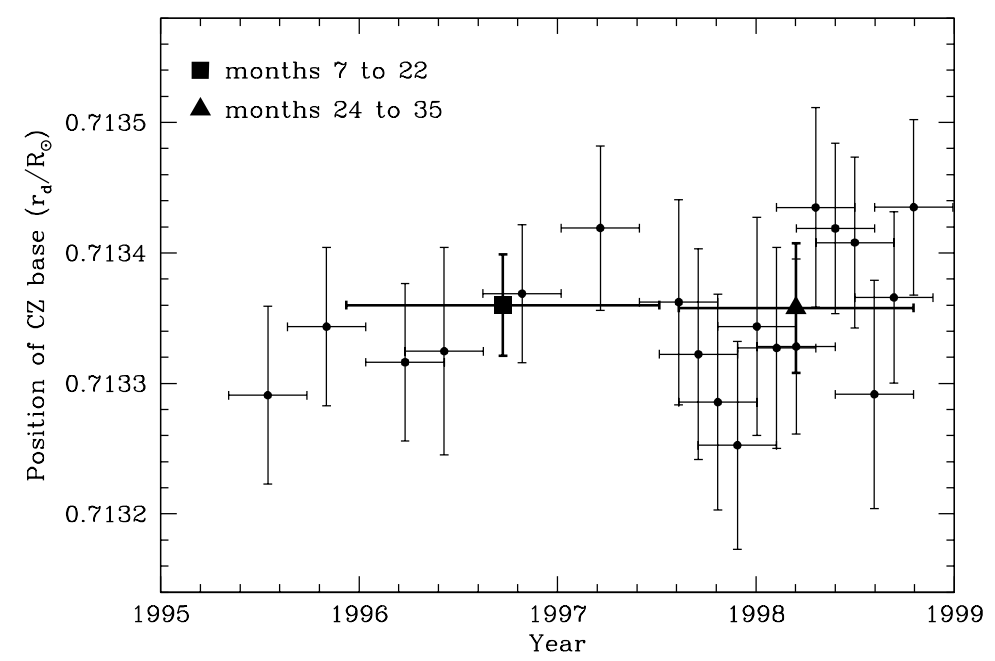

Figure 3. The position of the solar CZ base plotted as a function of time. Note that the error estimates do not include any contribution from systematic errors. These will be similar for all the results shown. The horizontal error-bars indicate the period of time over which the data were collected.

layers of the Sun. The frequency difference for months 8-11 which is close to solar minimum is negative, while those from months $1-4$, which is before the minimum in cycle 22 , is slightly positive. As we approach the solar maximum the frequency difference increases rapidly.

The change in solar oscillation frequencies is found to be well correlated with the activity indicators like the sunspot number. Fig. 2 shows the mean frequency shift averaged over all modes with $1.5 \leq \nu \leq 3.5$ $\mathrm{mHz}$ and $5 \leq \ell \leq 100$ which are common to all data sets, as a functions of time. This figure also shows the mean daily sunspot number, $R_{I}$ during the corresponding period as obtained from Solar Geophysical data web page (http://www.ngdc.noaa.gov/stp/stp.html of the US National Geophysical Data Center. The quantity $R_{I}$ is a measure of solar activity. It is quite clear that the two quantities are correlated. Similar figures can be drawn for other activity indices (Bhatnagar et al. 1999).

In order to study possible temporal variation of the internal structure of the Sun, we use the frequencies from each data set to calculate the position of the base of the convection zone $\left(r_{d}\right)$. The results from GONG data are shown as a function of time in Fig. 3. There is no systematic pattern to suggest that there are any solar cycle related changes in the CZ depth. Fig. 4 shows the same results but plotted as a function of the mean daily sunspot number, $R_{I}$. Once again there is no clear trend visible and all points appear to be consistent with 


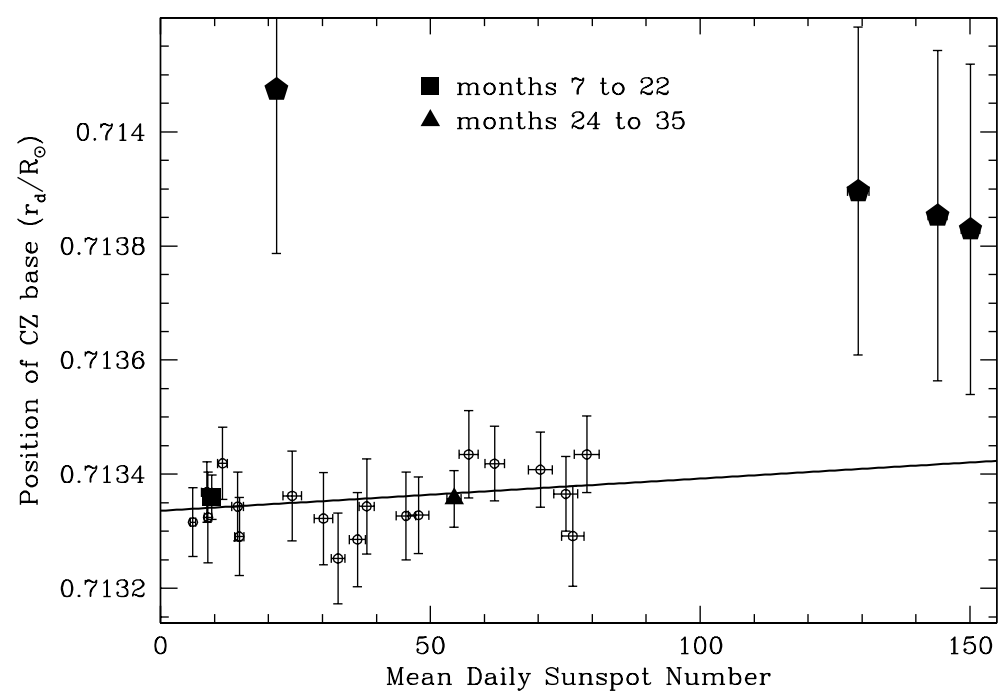

Figure 4. The position of the solar CZ base plotted as a function of the mean daily sunspot number. The large, filled pentagons are from the BBSO data taken during the last solar cycle. The straight line shows the best fit to GONG points.

the constant value found using summed spectra for months 7-22 or months 24-35. These values are more precise because it involves sum over more spectra covering a larger time interval. Again there is no significant difference between these two values. If we try to fit a straight line through the points from GONG data in Fig. 4, then the resulting slope is $(5.6 \pm 5.6) \times 10^{-7}$, which is hardly significant. More data from subsequent months with higher activity should be able to estimate this slope more reliably. The few results that we have from the previous solar cycle obtained by the Big Bear Solar Observatory (BBSO) also do not show any variation with solar activity, but the results are systematically larger than the GONG results. This difference could be due to systematic differences in the two data sets and may not represent any real changes in solar interior.

We also calculate the extent of overshoot below the convection zone using all the data sets. Fig. 5 shows how the amplitude of the oscillatory part in the fourth difference of the frequencies due to the transition at the base of the overshoot layer, changes as a function of the mean daily number of sunspots. The amplitude should increase with any increase in the extent of overshoot below the solar CZ base. The points corresponding to 4 month averaged spectra are systematically above the mean values determined from the spectra summed over the larger time intervals. This is because an increase in the errors of the frequencies, in general, tends to increase the estimated amplitude of the signal (Basu 


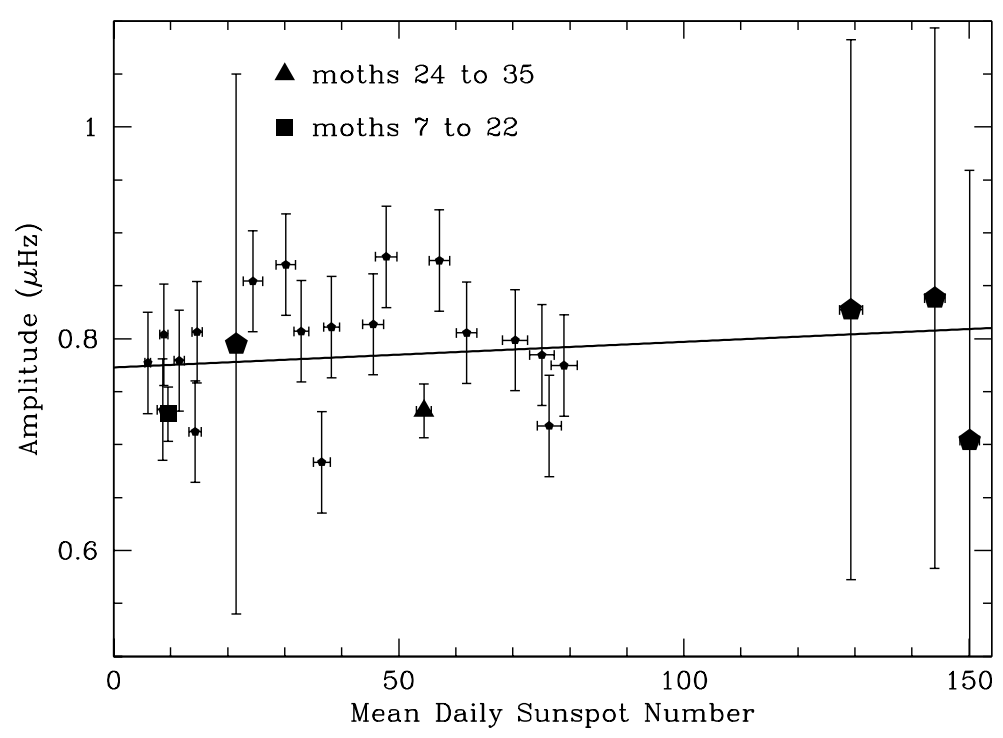

Figure 5. The amplitude of the oscillatory signal in the fourth difference of frequencies plotted as a function of the average, number of sunspots per day during the observing period. The large, filled pentagons are results from data obtained by BBSO during the last solar cycle. The straight line shows the best fit to GONG points.

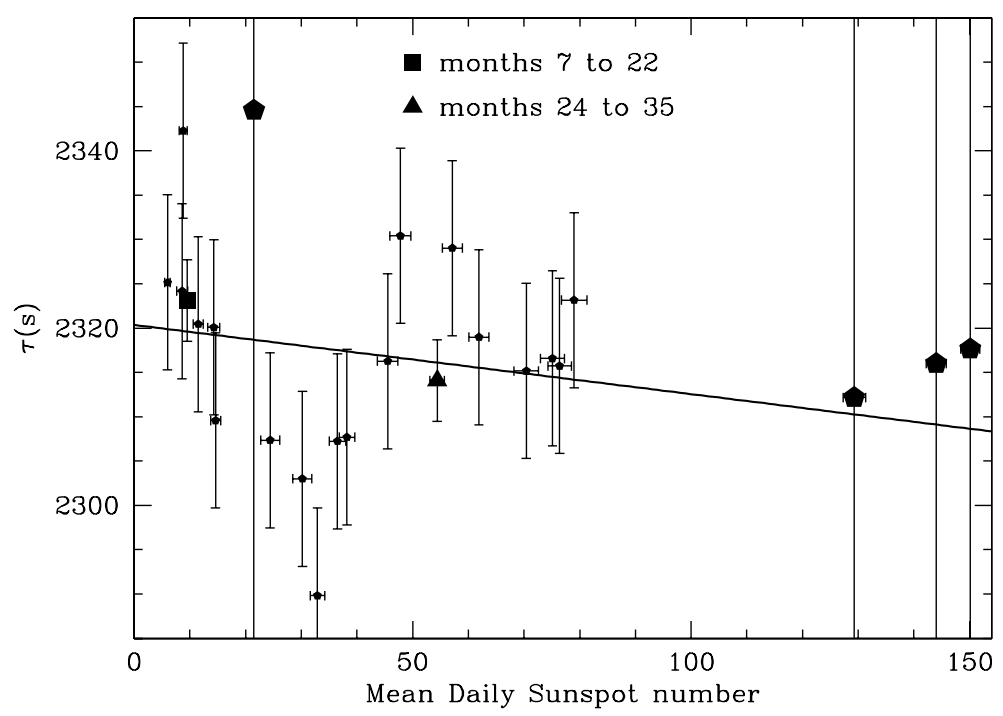

Figure 6. The frequency of the oscillatory signal in the frequencies plotted as a function of the average, daily number of sunspots. The large, filled pentagons are results from BBSO data taken during the last solar cycle. The straight line shows the best fit to GONG points. 
1997). Again we fail to find any systematic trend to indicate solar-cycle related changes in the extent of the overshoot layer. The BBSO results from the previous solar cycle are in good agreement with the GONG results. A straight line fit through the GONG points yields a slope of $(2.4 \pm 6.0) \times 10^{-4} \mu \mathrm{Hz}$, which is consistent with zero.

Fig. 6 shows how the frequency of the oscillatory part in frequencies due to the transition at the base of the overshoot layer changes as a function of the mean daily sunspot number. This frequency is expected to be the acoustic depth of the base of overshoot layer. Once again we do not see any systematic variation in the acoustic depth with time. A straight line fit through the GONG points yields a slope of $-0.078 \pm$ $0.077 \mathrm{~s}$, which is hardly significant. This is consistent with the fact that we do not find any significant variation in the $\mathrm{CZ}$ depth. The errors in estimated $\tau$ are much larger than those in estimating $\mathrm{CZ}$ depth directly and $\tau$ may have some uncertainty arising from those in surface layers. Some of the variation in $\tau$ could also be due to variation in surface layers. A variation in $\mathrm{CZ}$ depth by $0.0001 R_{\odot}$ will change $\tau$ by about $0.3 \mathrm{~s}$, which is too small to be detected. Change in extent of overshoot can also affect $\tau$ but once again the amplitude of oscillatory signal is more sensitive to these changes. Hence the variation, if any, in $\tau$ is likely to be due to surface effects.

Having failed to find any systematic change in the depth of the convection zone or the extent of overshoot below the solar convection zone, we attempt to see if there are any significant changes taking place in the solar interior. To test this we take the frequency difference between months 32-35 and 7-22, and apply inversion techniques to see if there is any corresponding change in the sound-speed profile inside the Sun. We have chosen these two sets since solar activity changed significantly between these two intervals, thereby causing a large change in frequencies. For other pairs of data sets the frequency difference is smaller. Fig. 7 shows the relative sound speed differences that result from frequency differences between months 32-35 and 7-22 using various inversion techniques. We have tried the asymptotic inversion technique (Christensen-Dalsgaard, Gough and Thompson 1989) as well as nonasymptotic inversion using the Regularized Least Squares (RLS) technique as described by Antia (1996) and the Subtractive Optimally Localized Averages (SOLA) technique as described by Basu et al. (1996). All these inversion results agree with each other and the resulting variation in sound speed is within the expected errors. These results confirm our expectation that the frequency difference probably do not imply changes in structure deep inside the Sun. similar results have been found for frequency difference between other pairs of data sets. Thus it appears that the changes in frequency with time are due 


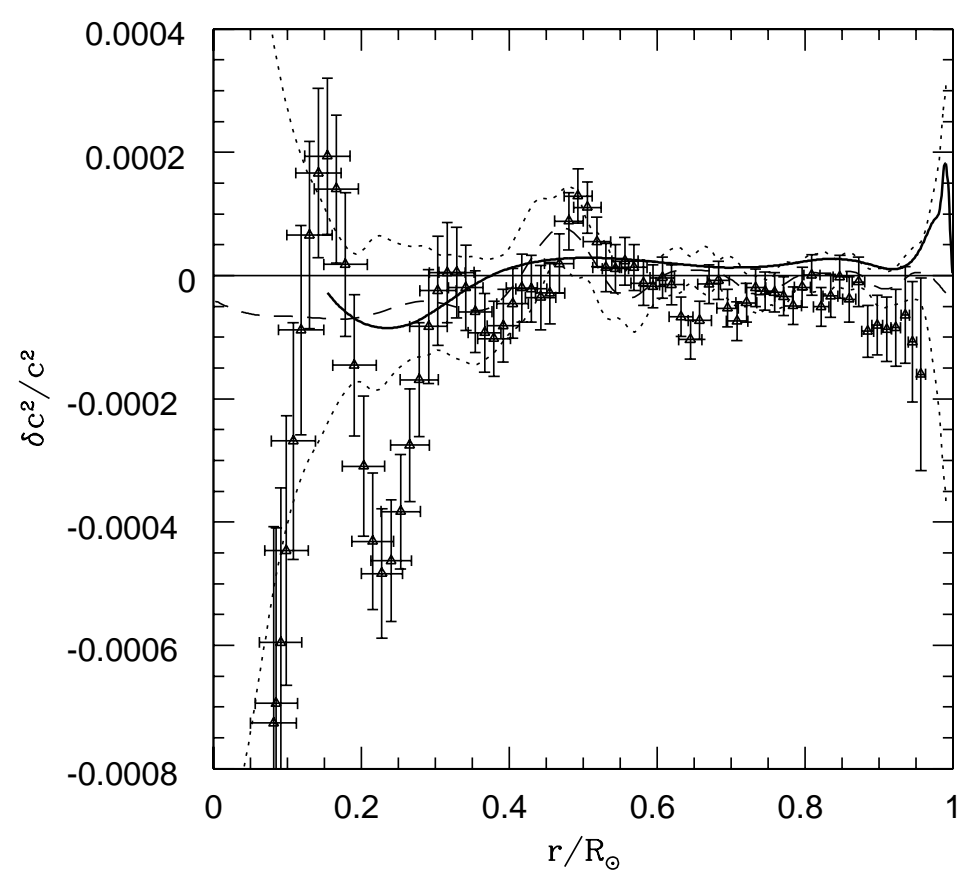

Figure \%. The relative sound-speed differences that result from the difference in frequencies between months $32-35$ and $7-22$. The points are the result of an Subtractive Optimally Localized Averages (SOLA) inversion. The dashed line with the dotted lines showing the $1 \sigma$ error limits are results of a Regularized Least Squares (RLS) inversion. The thick continuous line is a result using an asymptotic inversion.

to variations in surface layers, which do not affect the deep interior significantly.

\section{Conclusions}

The frequencies of solar oscillations show a variation which is correlated with solar activity. We find no clear evidence of solar cycle related change in the depth of the convection zone or the extent of overshoot below the convection zone. Our study has been mainly restricted to the rising phase of the cycle 23 and it would be interesting to check the variations in subsequent part of the solar cycle. Within the period of 1995 to 1998 considered in this study, the changes, if any, in the depth of the $\mathrm{CZ}$ should be less than the error limits in our estimate, i.e., $0.0001 R_{\odot} \approx 70 \mathrm{~km}$. There is no significant change in the sound speed in deep interior. Thus it appears that solar cycle changes in solar structure are predominantly confined to near surface regions. 


\section{Acknowledgements}

This work utilizes data obtained by the Global Oscillation Network Group (GONG) project, managed by the National Solar Observatory, a Division of the National Optical Astronomy Observatories, which is operated by AURA, Inc. under a cooperative agreement with the National Science Foundation. The data were acquired by instruments operated by the Big Bear Solar Observatory, High Altitude Observatory, Learmonth Solar Observatory, Udaipur Solar Observatory, Instituto de Astrofisico de Canarias, and Cerro Tololo Inter-American Observatory.

\section{References}

Anderson, E. R., Duvall, T. L., Jr., and Jefferies, S. M.: 1990, Astrophys. J. 364, 699.

Antia, H. M.: 1996, Astron. Astrophys. 307, 609.

Basu, S.: 1997, Mon. Not. Roy. Astron. Soc. 288, 572.

Basu, S., and Antia, H. M.: 1997, Mon. Not. Roy. Astron. Soc. 287, 189.

Basu, S., and Antia, H. M.: 2000, Astrophys. J. (in press) astro-ph/9911007

Basu, S., Antia, H. M., and Narasimha, D.: 1994, Mon. Not. Roy. Astron. Soc. 267, 209.

Basu, S, Christensen-Dalsgaard, J., Pérez Hernández, F., and Thompson, M, J.: 1996, Mon. Not. Roy. Astron. Soc. 280, 651.

Bhatnagar, A., Jain, K., and Tripathy, S. C.: 1999, Astrophys. J. 521, 885.

Christensen-Dalsgaard, and J., Berthomieu, G.: 1996, in Solar Interior and Atmosphere, eds/ A. N. Cox et al., Space Science Series, (Tucson: University of Arizona Press) p401.

Christensen-Dalsgaard, J., Gough, D. O., and Thompson, M. J.: 1989, Mon. Not. Roy. Astron. Soc. 238, 481.

Dziembowski, W. A., Goode, P. R., DiMauro, M. P., Kosovichev, A. G., and Schou, J.: 1998, Astrophys. J. 509, 456.

Elsworth, Y., Howe, R., Isaak, G. R., McLeod, C. P., and New, R.: 1990, Nature $\mathbf{3 4 5}, 322$.

Gough, D. O.: 1990, in Osaki, Y., and Shibahashi, H., eds., Lecture Notes in Physics, 367, Springer, Berlin, p.283.

Howe, R., Komm, R., and Hill, F.: 1999, Astrophys. J. 524, 1084.

Libbrecht, K. G., and Woodard, M. F.: 1990, Nature 345, 779.

Monteiro, M. J. P. F. G., Christensen-Dalsgaard, J., and Thompson, M. J.: 1994, Astron. Astrophys. 283, 247.

Monteiro, M. J. P. F. G., Christensen-Dalsgaard, J., and Thompson, M. J.: 1998, in Proc: SOHO6/GONG98 workshop, Structure and Dynamics of the Interior of the Sun and Sun-like Stars, eds. S. Korzennik, A. Wilson, ESA SP-418 (ESA: Noordwijk), p 495.

Woodard, M., and Libbrecht, K. G.: 1993, Astrophys. J. 402, L77. 